\title{
Periodic and chaotic solutions in infinite dimensional systems
}

\author{
Michal Fečkan
}




\title{
PERIODIC AND CHAOTIC SOLUTIONS IN INFINITE DIMENSIONAL SYSTEMS
}

\author{
MiChAL FEČKAN \\ Department of Mathematical Analysis, Comenius University \\ Mlynská dolina, 84248 Bratislava, Slovakia \\ Michal.Feckan@fmph.uniba.sk
}

[Received June 20, 2000]

\begin{abstract}
Periodic, subharmonic and chaotic weak solutions are investigated for certain damped and undamped forced nonlinear hyperbolic partial differential equations of a beam.

Mathematical Subject Classification: 35B10, 35B20

Keywords: Periodic and chaotic solutions, weak solutions, infinite dimensional systems
\end{abstract}

\section{Introduction}

This note is a survey of our recent results $[3,4,7]$. We here focus on concrete problems and we refer the reader for proofs of results and for an abstract setting of problems to the above mentioned papers.

P. Holmes and J. Marsden in [10] studied a forced partial differential equation of a beam of the form

$$
\begin{gathered}
w_{t t}+w_{x x x x}+\Gamma w_{x x}-\kappa\left(\int_{0}^{1} w_{x}^{2}(s, t) d s\right) w_{x x}=\epsilon\left(q \cos \omega t-\delta w_{t}\right) \\
w(0, \cdot)=w(1, \cdot)=w_{x x}(0, \cdot)=w_{x x}(1, \cdot)=0,
\end{gathered}
$$

where $\Gamma=$ external load, $\kappa=$ stiffness due to "membrane" effect, $\delta=$ damping, and $\epsilon$ is small. They shown the existence of a Smale horseshoe. Consequently, (1.1) possesses an infinite number of subharmonic weak solutions. The new way in this direction is established in a series of papers by D.W. McLaughlin, J. Shatah, E.A. Overman II, S. Wiggins, C. Xiong and Y. Li [14-16]. They studied perturbed nonlinear Schrödinger equations and perturbed sine-Gordon equations.

We study in [3] a forced sine-beam partial differential equation given by

$$
\begin{gathered}
u_{t t}+\alpha u_{x x x x}+\sin u+\tau(x)=\epsilon \sin t \\
u(x+1, t)=u(x, t)
\end{gathered}
$$

where $\alpha>0$ is a constant, $\tau$ is continuous, 1 -periodic satisfying $\int_{0}^{1} \tau(x) d x=0$ and $\epsilon \in \mathbf{R}$ is a small parameter. 
A modified version of (1.1) is investigated in [4] of the form

$$
\begin{gathered}
u_{t t}+u_{x x x x}+\Gamma u_{x x}+p\left(\int_{0}^{1} u^{2}(s, t) d s, \int_{0}^{1} u_{x}^{2}(s, t) d s\right) D_{x x}^{\xi} u=\epsilon q(x) \cos \frac{2 \pi t}{T} \\
u(0, \cdot)=u(1, \cdot)=u_{x x}(0, \cdot)=u_{x x}(1, \cdot)=0
\end{gathered}
$$

where $D_{x x} u=-u_{x x}, D_{x x}^{\xi}$ is the $\xi$-power of $D_{x x}$ in $L^{2}(0,1), 0 \leq \xi \leq 1, \Gamma \in \mathbf{R}$, $p \in C^{2}(\mathbf{R} \times \mathbf{R}, \mathbf{R}), p(0,0)=0, q \in H^{2}(0,1) \cap H_{0}^{1}(0,1), T>0$ and $\epsilon \in \mathbf{R}$ is a small parameter.

We assume that $\alpha$ is sufficiently large and also consider a limit ordinary differential equation of (1.2) as $\alpha \rightarrow \infty$ of the form

$$
\ddot{u}+\sin u=\epsilon \sin t .
$$

It is well-known $[8,18]$ that for $\epsilon \neq 0$ sufficiently small, (1.4) exhibits a chaotic behaviour. In particular, it has an infinite number of subharmonic librational solutions with periods tending to infinity.

Similarly, if we put

$$
u(x, t)=z(t) \sqrt{2} \sin \pi x
$$

in $(1.3)$ with $\epsilon=0[10,11]$ we arrive at an ordinary differential equation

$$
\ddot{z}+\pi^{2}\left(\pi^{2}-\Gamma\right) z+p\left(z^{2}, \pi^{2} z^{2}\right) \pi^{2 \xi} z=0 .
$$

By assuming

$$
\Gamma>\pi^{2}, \quad p\left(z, \pi^{2} z\right)=\kappa z \quad \forall z \geq 0
$$

for a constant $\kappa>0,(1.5)$ becomes the Duffing equation [8]. It is well-known that the Duffing equation possesses a one-parametric bounded family of periodic solutions with periods tending to infinity.

We note that (1.2) and (1.3) are undamped and (1.1) is damped for $\epsilon \neq 0$. For this reason, we show in $[3,4]$ only the existence of finitely, but arbitrarily many subharmonic weak solutions of (1.2) or (1.3) persisting from (1.4) or (1.5) when $\alpha \rightarrow \infty$ or $\epsilon$ is sufficiently small and $\int_{0}^{1} q(x) \sin \pi x d x \neq 0$, respectively.

A damped case of (1.1) is studied in [7] of the following form: The boundary value problem for planar deflections of an elastic beam with a compressive axial load $\Gamma$ and pinned ends is

$$
\begin{gathered}
\ddot{u}=-u^{\prime \prime \prime \prime}-\Gamma u^{\prime \prime}+\left[\int_{0}^{\pi}\left(u^{\prime}(s)\right)^{2} \mathrm{~d} s\right] u^{\prime \prime}-2 \mu_{2} \dot{u}+\mu_{1} \cos \omega_{0} t, \\
u(0, t)=u(\pi, t)=u^{\prime \prime}(0, t)=u^{\prime \prime}(\pi, t)=0,
\end{gathered}
$$

where $u(x, t)$ is the transverse deflection at a distance $x$ from one end at time $t$. In (1.7), a superior dot denotes differentiation with respect to $t$ and prime differentiation with respect to $x$. We consider the $\mu_{i}$ terms as perturbations. 
Our first step is to consider the linearized, unperturbed problem and compute the eigenvalues at the origin which are $\lambda_{n}=n^{2}\left(n^{2}-\Gamma\right)$ and corresponding eigenfunctions $\varphi_{n}(x)=\sin n x$ for $n=1,2, \cdots$. For small $\Gamma$ the origin is a center with the first bifurcation occurring at $\Gamma=1$, the first Euler buckling load. The corresponding eigenfunction, $\varphi_{1}(x)=\sin x$, is referred to as the first buckled mode. When $\Gamma$ is sufficiently large, (1.7) can exhibit chaotic behavior. The first work on this was done by P. Holmes [9] and extended by P. Holmes and J. Marsden [10, 11]. Some more recent work on the full equation is by H.M. Rodrigues and M. Silveira [19], S.W. Shaw [21] and by M. Berti and C. Carminati [2].

In (1.7) substitute $u(x, t)=\sum_{k=1}^{\infty} u_{k}(t) \sin k x$, multiply by $\sin n x$ and integrate from 0 to $\pi$. This yields the infinite set of ordinary differential equations

$$
\begin{gathered}
\ddot{u}_{n}=n^{2}\left(\Gamma-n^{2}\right) u_{n}-\frac{\pi}{2} n^{2}\left[\sum_{k=1}^{\infty} k^{2} u_{k}^{2}\right] u_{n}-2 \mu_{2} \dot{u}_{n}+2 \mu_{1} \cos \omega_{0} t \\
n=1,2, \cdots .
\end{gathered}
$$

We see that the linear parts of these equations are uncoupled and the equations divide into two types. The system of equations where $1 \leq n^{2}<\Gamma$ has a hyperbolic equilibrium at the origin whereas, for $n^{2} \geq \Gamma$ this equilibrium is a center. Infinite sets of ordinary differential equations are also investigated in [20].

For simplicity let us assume $1<\Gamma<4$. Then only the equation with $n=1$ is hyperbolic while the remaining equations have a center. To emphasize this let us define $p=u_{1}$ and $q_{n}=u_{n+1}, n=1,2, \cdots$. The preceding equations now take the form

$$
\begin{gathered}
\ddot{p}=a^{2} p-\frac{\pi}{2}\left[p^{2}+\sum_{k=1}^{\infty}(k+1)^{2} q_{k}^{2}\right] p-2 \mu_{2} \dot{p}+\frac{4}{\pi} \mu_{1} \cos \omega_{0} t, \\
\ddot{q}_{n}=-\omega_{n}^{2} q_{n}-\frac{\pi}{2}(n+1)^{2}\left[p^{2}+\sum_{k=1}^{\infty}(k+1)^{2} q_{k}^{2}\right] q_{n} \\
-2 \mu_{2} \dot{q}_{n}+2 \mu_{1}\left[\frac{1-(-1)^{n+1}}{\pi(n+1)}\right] \cos \omega_{0} t, \quad n=1,2, \cdots,
\end{gathered}
$$

where we have defined $a^{2}=\Gamma-1$ and $\omega_{n}^{2}=(n+1)^{2}(n+1)^{2}-\Gamma \operatorname{In}(1.8)$ we project onto the hyperbolic subspace by setting $q=0$ in the first equation of (1.8) to obtain what we shall call the reduced equation. In our example this is

$$
\ddot{p}=a^{2} p-\frac{\pi}{2} p^{3}-2 \mu_{2} \dot{p}+\frac{4}{\pi} \mu_{1} \cos \omega_{0} t .
$$

We see that this is the forced, damped Duffing equation with negative stiffness for which standard theory yields chaotic dynamics. The purpose of [7] is to show that 
the chaotic dynamics of (1.9) are, in some sense, shadowed in the dynamics of the full equation (1.8).

\section{The problem (1.2)}

By using the results of $[18$, p. 253], we know that $(-\pi, 0)$ and $(\pi, 0)$ are hyperbolic fixed points of the ordinary differential equation

$$
\dot{x}=y, \quad \dot{y}=-\sin x
$$

joined by the upper separatrix

$$
(\gamma, \dot{\gamma}), \quad \gamma=\pi-4 \tan ^{-1}\left(\mathrm{e}^{-t}\right)
$$

We consider (1.4) as an ordinary differential equation on the circle $S^{2 \pi}=\mathbf{R} /[0,2 \pi]$. Then (2.1) is defined on the cylinder $S^{2 \pi} \times \mathbf{R} \ni(x, y)$ and $(-\pi, 0),(\pi, 0)$ are glued to a hyperbolic fixed point of (2.1) joined by the homoclinic orbit $(\gamma, \dot{\gamma})$.

We note that $\lambda_{k}=16 \pi^{4} k^{4}, k \in \mathbf{Z}_{+}=\{0\} \cup \mathbf{N}$ are eigenvalues of $u_{x x x x}$ with the periodic boundary value condition of (1.2), and

$$
\sum_{\lambda_{k}>0}\left(\frac{3}{\sqrt{\lambda_{k}}}+\frac{1}{\lambda_{k}}\right)=\sum_{m \geq 1}\left(\frac{3}{4 \pi^{2} m^{2}}+\frac{1}{16 \pi^{4} m^{4}}\right)=\frac{181}{1440} .
$$

We need the following lemma.

Lemma 2.1. ([3]) Let $D \in \mathbf{N}$. If $S(d)$ is the set of all $\omega \geq 1$ satisfying

$$
\left|\omega-\frac{n}{4 \pi^{2} k^{2}}\right| \geq \frac{d}{16 \pi^{4} k^{4}} \quad \forall k>0, \forall n \in \mathbf{Z}_{+}
$$

with $0<d<16 \pi^{4}$, then

$$
m(S(d) \cap[D, D+1]) \geq 1-\frac{181}{720} d,
$$

where $m(\cdot)$ is the Lebesque measure.

Now we can state the main result of [3] concerning (1.2).

Theorem 2.2. ([3]) Let $8>\rho>5$ and $0<d<720 / 181$. There are constants $K_{1}>0, K_{2}>0$ such that for any $1>|\epsilon|>0, m \in \mathbf{N}, 0<\eta \leq 1$ satisfying

$$
\frac{1}{|\epsilon|^{1 / 2}}<m<\frac{K_{1}}{2 \pi|\epsilon|^{(\rho-4) / 2}}, \quad \frac{m}{\eta|\epsilon|^{\rho / 2}} \in S(d),
$$

(1.2) has a $2 \pi m$-periodic weak solution $u_{m}$ with $\alpha=\frac{1}{\eta^{2}|\epsilon|^{\rho}}$ satisfying

$$
\max _{-\pi m \leq t \leq \pi m}\left|\int_{0}^{1} u_{m}(x, t) d x-\gamma\left(t-\delta_{m}\right)\right| \leq K_{2}|\epsilon|
$$


for some $\delta_{m} \in\left[-K_{2}, K_{2}\right]$.

The above results can be modified also to the equations

$$
\begin{gathered}
u_{t t}+\alpha u_{x x x x}+\sin u+\tau(x)=\epsilon \cos t, \quad u(x, t)=u(1-x, t) \\
u_{x x}(0, t)=u_{x x}(1, t)=u_{x x x}(0, t)=u_{x x x}(1, t)=0,
\end{gathered}
$$

and

$$
\begin{gathered}
u_{t t}+\alpha u_{x x x x}+2 u^{3}-u+\tau(x)=\epsilon \cos t \\
u(x+1, t)=u(x, t)
\end{gathered}
$$

where $\alpha>0$ is a constant, $\tau$ is continuous satisfying $\int_{0}^{1} \tau(x) d x=0$ and $\epsilon \in \mathbf{R}$ is a small parameter. Moreover, $\tau$ is either symmetric with respect to $x=1 / 2$ for (2.2) or 1 -periodic for (2.3). We note that the limit equation of (2.3) as $\alpha \rightarrow \infty$ is the forced Duffing equation given by

$$
\ddot{u}+2 u^{3}-u=\epsilon \cos t .
$$

We end this section with an existence result following directly from [3, Theorem 2.9].

Theorem 2.3. Let $0<d<720 / 181$. The equation

$$
\begin{gathered}
u_{t t}+\alpha u_{x x x x}+u+\theta \sin u+\tau(x)=\epsilon \sin t \\
u(x+1, t)=u(x, t),
\end{gathered}
$$

where $\tau$ is the same as in (1.2) and $0<\theta<1$, has a unique $2 \pi$-periodic weak solution for any $\epsilon \in \mathbf{R}$ and $\alpha$ satisfying

$$
\alpha>(1+)^{2} / d^{2} \quad \text { and } \quad \sqrt{\alpha} \in S(d) .
$$

\section{The problem (1.3)}

In this section we recall some results of [4] concerning (1.3). We note that

$$
\lambda_{j}=\pi^{2} j^{2}\left(\pi^{2} j^{2}-\Gamma\right), \quad j \in \mathbf{N}
$$

are the eigenvalues of the linear part of (1.3) given by $u_{x x x x}+\Gamma u_{x x}$ with the corresponding boundary value conditions.

Lemma 3.1. ([4]) Let $D \geq 0$ and $\rho>1 / 4$. If $S(c)$ is the set of all $T>0$ satisfying

$$
\lambda_{j}^{\rho}\left|\sin \frac{\sqrt{\lambda_{j}} T}{2}\right| \geq c \quad \forall j \in \mathbf{N}: j^{2}>\Gamma / \pi^{2},
$$


where $c>0$ is a constant, then the Lebesque measure of the complement

$$
(\mathbf{R} \backslash S(c)) \cap[D, D+1]
$$

satisfies

$$
m((\mathbf{R} \backslash S(c)) \cap[D, D+1]) \leq \sum_{k^{2}>\Gamma / \pi^{2}}\left(\frac{2 c \pi}{\sqrt{\lambda_{k}} \lambda_{k}^{\rho}}+\frac{c}{\lambda_{k}^{\rho}}+\frac{2 c^{2} \pi}{\sqrt{\lambda_{k}} \lambda_{k}^{2 \rho}}\right) .
$$

Now we can state the main result of [4] concerning (1.3).

Theorem 3.2. ([4]) Consider (1.3) with $0 \leq \xi \leq 1, \pi^{2}<\Gamma<3 \pi^{2}$. Let $1 \geq c>0$ and let (1.6) hold. If $\int_{0}^{1} q(x) \sin \pi x d x \neq 0$ then for any $p \in \mathbf{N}, T$ satisfying

$$
\frac{\sqrt{2}}{\sqrt{\Gamma-\pi^{2}}} \leq p T<\frac{1}{4^{\xi} \sqrt{8+2\left(4 \pi^{4}\right)^{1-\xi}}} \frac{c}{\Gamma-\pi^{2}}
$$

and $p T \in S(c)$, the equation (1.3) has a $p T$-periodic weak solution for any $\epsilon$ suffciently small. Moreover, if $0 \leq \xi<1 / 2$, then $m(S(c) \cap[D, D+1]) \rightarrow 1$ as $c \rightarrow 0_{+}$ uniformly with respect to $D \geq 0$.

Roughly speaking, Theorem 3.2 asserts that (1.3) with $0 \leq \xi<1 / 2$ and $\pi^{2}<\Gamma<$ $3 \pi^{2}$, has many subharmonics for $\epsilon$ sufficiently small when $c$ and $\pi^{2}-\Gamma$ are sufficiently small. On the other hand, since we do not know the structure of the set $S(c)$, we do not know a concrete case for $p T$. This result can be related to the KAM theory [13]. Furthermore, Lemma 3.1 gives that the Lebesque measure of $S(c)$ tends to 1 as $c \rightarrow 0_{+}$. We note [4] that now $\rho=(1-\xi) / 2$ in Lemma 3.1. Consequently, we put $c=\left(\Gamma-\pi^{2}\right)^{1 / 4}$ in Theorem 3.2, and the assumptions of Theorem 3.2 take the following form

$$
\frac{\sqrt{2}}{\sqrt{\Gamma-\pi^{2}}} \leq p T<\frac{1}{4 \xi \sqrt{8+2\left(4 \pi^{4}\right)^{1-\xi}}} \frac{1}{\left(\Gamma-\pi^{2}\right)^{3 / 4}}, \quad p T \in S\left(\left(\Gamma-\pi^{2}\right)\right) .
$$

Summarizing we obtain the following result.

Theorem 3.3. ([4]) Let $1 / 2>\xi \geq 0$ and $\pi^{2}<\Gamma<3 \pi^{2}$. If (1.6) holds and $\int_{0}^{1} q(x) \sin \pi x d x \neq 0$, then the smaller $\Gamma-\pi^{2}>0$, the larger number of subharmonic weak solutions of (1.5) persists for (1.3) when $\epsilon$ is sufficiently small.

The above results deal with the bifurcation of subharmonic weak solutions. Now we formulate some results about the existence of periodic weak solutions for (1.3).

Theorem 3.4. ([4]) Let $1 / 2>\xi \geq 0$ and $\Gamma \in \mathbf{R} \backslash\left\{\pi^{2} j^{2} \mid j \in \mathbf{N}\right\}$ be arbitrari. For almost all $T>0$ (in the sense of the Lebesque measure), there is a small $T$-periodic weak solution of (1.3) for any $\epsilon$ sufficiently small.

We do not know the form of $T$ in the above result. Particular results with concrete $T$ and $\Gamma$ are given bellow. Related results are proved in $[1,12,17,22,23]$. 
Theorem 3.5. ([4]) Consider (1.3) with $1 \geq \xi \geq 0$. If $T \pi \in \mathbf{Q}$ and $\Gamma / \pi^{2} \notin \mathbf{Q}$, then there is a small $T$-periodic weak solution of (1.3) for any $\epsilon$ sufficiently small.

Theorem 3.6. ([4]) Consider (1.3) with $\xi=0$. If $T=2 \Omega / \pi, \Gamma=2 m \pi^{2} / \Omega$, where $m \in\{-1,0\}$ and $\Omega>0$ has the infinite continuous fraction decomposition [17] $\Omega=\left[a_{0}, a_{1}, a_{2}, \cdots\right]$ such that $a_{i} \leq M \forall i \in \mathbf{Z}_{+}$for a constant $M \in \mathbf{N}$ satisfying $m^{2}(M+2)<2 \Omega$, then there is a small $T$-periodic weak solution of (1.3) for any $\epsilon$ sufficiently small.

We remark that Theorem 3.4 provides a more general result than Theorems 3.5 and 3.6, while Theorems 3.5 and 3.6 give concrete forms of $T$ and $\Gamma$ for the existence of periodic solutions of (1.3). For instance, Theorem 3.6 is valid for $\Omega=$ $\left(M+\sqrt{M^{2}+4 M}\right) / 2, M \in \mathbf{N}$ and $m \in\{-1,0\}$.

Results like above are obtained in [4] also for the equation

$$
\begin{gathered}
u_{t t}+u_{x x x x}+\Gamma u_{x x}+p\left(\int_{0}^{2} u^{2}(s, t) d s, \int_{0}^{2} u_{x}^{2}(s, t) d s\right) D_{x x}^{\xi} u \\
=\epsilon\left(q_{1}(x) \cos \frac{2 \pi t}{T}+q_{2}(x) \sin \frac{2 \pi t}{T}\right), \\
u(x, \cdot)=u(x+2, \cdot) \quad \forall x \in \mathbf{R},
\end{gathered}
$$

where $\xi, \Gamma, p$ are like in $(1.3), D_{x x}^{\xi}$ is the $\xi$-power of $D_{x x}$ in $L^{2}(\mathbf{R} / 2 \mathbf{Z}), q_{1,2} \in H^{2}(0,2)$ and $q_{1,2}$ are 2 -periodic satisfying

$$
\begin{array}{ll}
\int_{0}^{2} q_{1}(x) \cos \pi x d x=0, & \int_{0}^{2} q_{2}(x) \sin \pi x d x=0, \\
\int_{0}^{2} q_{1}(x) \sin \pi x d x \neq 0, & \int_{0}^{2} q_{2}(x) \cos \pi x d x \neq 0 .
\end{array}
$$

\section{The problem (1.7)}

The reduced equations of (1.9) are

$$
\begin{aligned}
& \dot{x}_{1}=x_{2}, \\
& \dot{x}_{2}=a^{2} x_{1}-\frac{\pi}{2} x_{1}^{3}-2 \mu_{2} x_{2}+\frac{4}{\pi} \mu_{1} \cos \omega_{0} t
\end{aligned}
$$

obtained by setting $q=0$ in the hyperbolic part of (1.8). When $\mu=0$, (4.1) has a homoclinic solution given by $\gamma=(r, \dot{r})$ where $r(t)=(2 a / \sqrt{\pi}) \operatorname{sech} a t$. The Melnikov homoclinic function $M$ for $(4.1)[7,18]$ becomes

$$
M(\mu, \alpha)=\left[\frac{8 \omega_{0}}{\sqrt{\pi}} \sin \omega_{0} \alpha \operatorname{sech} \frac{\pi \omega_{0}}{2 a}\right] \mu_{1}-\frac{16 a^{3}}{3 \pi} \mu_{2} .
$$

Thus, the conditions $M\left(\mu_{0}, \alpha_{0}\right)=0,(\partial M / \partial \alpha)\left(\mu_{0}, \alpha_{0}\right) \neq 0$ are satisfied for all $\mu_{0}$ such that

$$
\left|\frac{\mu_{0,2}}{\mu_{0,1}}\right|<\frac{3 \sqrt{\pi} \omega_{0}}{2 a^{3}} \operatorname{sech} \frac{\pi \omega_{0}}{2 a} .
$$


We now obtain the following result from [7].

Theorem 4.1. There exists a constant $C \geq 0$ so that whenever $\mu_{0}$ satisfies $C \leq\left|\frac{\mu_{0,2}}{\mu_{0,1}}\right|<\frac{3 \sqrt{\pi} \omega_{0}}{2 a^{3}} \operatorname{sech} \frac{\pi \omega_{0}}{2 a}$ there exists a corresponding $\bar{\xi}_{0}>0$ such that if $0<\xi \leq \bar{\xi}_{0}$, if the parameters in (1.7) are given by $\mu=\xi \mu_{0}$ and $\mu_{0,2} \neq 0$ then (1.7) is chaotic. In the nonresonant case, i.e. if $\omega_{0} \neq \omega_{n}$ for all $n$, then $C=0$.

It is interesting to look at some history of this problem. The first work was by P. Holmes [9] in which he started with the partial differential equation and carried out the Galerkin expansion but restricted his analysis to the reduced equation. The significance of that work is that it introduced the idea of Melnikov analysis. In subsequent work [10, 11] P. Holmes and J. Marsden extended the results to infinite dimension but abandoned the Galerkin approach in favor of nonlinear semigroup techniques directly in infinite dimensions. In our work [7] we go back to the original, simpler analysis of the reduced equation and then show that the results apply to the original partial differential equation. Some advantages to this are that the Galerkin projection is a technique familiar to many engineers and physicists and, also, we are able to utilize general Melnikov results in Section 3 of [7] and Palmer's important results of [18]. This is illustrated further in [7] for the following generalizations:

- Nonplanar motion of a symmetric beam with one buckled mode.

- Nonplanar, nonsymmetric beam with one buckled mode in each plane.

- Planar motion of a symmetric beam with multiple buckled modes.

Acknowledgement: Partially supported by Grant GA-MS 1/6179/00. This paper is in final form and no version of it will be submitted for publication elsewhere.

\section{REFERENCES}

[1] Ben-Naoum, A. K. and Mawhin, J.: The periodic-Dirichlet problem for some semilinear wave equations, J. Differential Equations 96, (1992), 340-354.

[2] Berti, M. and Carminati, C.: Chaotic dynamics for perturbations of infinite dimensional Hamiltonian systems, ISAS - International School for Advanced Studies, Technical Report (Ref. SISSA 65/99/M), 1999.

[3] FeČKan, M.: Periodic oscillations of abstract wave equations, J. Dyn. Diff. Equations, 10, (1998), 605-617.

[4] FEČKAN, M.: Bifurcation of periodics and subharmonics in abstract nonlinear undamped wave equations, J. Differential Equations, 153, (1999), 41-60.

[5] FeČKan, M.: Periodic solutions of certain abstract wave equations, Proc. Amer. Math. Soc., 123, (1995), 465-470.

[6] FeČKAn, M.: On the existence of periodic solutions for a certain type of nonlinear equations, J. Differential Equations, 121, (1995), 28-41.

[7] GRUENDLER, J. and FEČKAn, M.: The existence of chaos in infinite dimensional systems, preprint (2000).

[8] Guckenheimer, J. and Holmes, P.: Nonlinear Oscillations, Dynamical Systems, and Bifurcations of Vector Fields, Springer-Verlag, New York, 1983. 
[9] Holmes, P.: A nonlinear oscillator with a strange attractor, Phil. Trans. Roy. Soc. A, 292, (1979), 419-448.

[10] Holmes, P. and Marsden, J.: A partial differential equation with infinitely many periodic orbits: chaotic oscillations of a forced beam, Arch. Rational Mech. Anal., 76, (1981), 135-165.

[11] Holmes, P. and Marsden, J.: Bifurcation to divergence and flutter in flow-induced oscillations: an infinite dimensional analysis, Automatica, 14, (1978), 367-384.

[12] Kolesov, A. Yu., Mischehenko, E. F. and Rozov, N. Kh.: Asymptotic Methods of Investigation of Periodic Solutions of Nonlinear Hyperbolic Equations, Proc. V.A. Steklov Inst. Math. 222, Nauka Publ.-MAIK Nauka, Moscow, 1998 (in Russian).

[13] Kuksin, S. B.: Nearly Integrable Infinite-Dimensional Hamiltonian Systems, Lec. Not. Math. Vol. 1556, Springer-Verlag, Berlin, 1993.

[14] McLaughlin, D.W. and Overman II, E.A.: Whiskered tori for integrable pde's: chaotic behavior in near integrable pde's, Surveys Appl. Math., 1, (1995), 83-203.

[15] Mclaughlin, D.W., Overman II, E.A., Wiggins, S. and XIOng, C.: Homoclinic orbits in a four-dimensional model of a perturbed NLS equation: a geometric singular perturbed study, Dynamics Reported, (1995), 190-287.

[16] Mclaughlin, D.W. and Shatan, J.: Homoclinic orbits for pde's, preprint (1997).

[17] McKenna, P. J.: On solutions of a nonlinear wave equation when the ratio of the period to the length of the interval is irrational, Proc. Amer. Math. Soc., 93, (1985), 59-64.

[18] Palmer, K. J.: Exponential dichotomies and transversal homoclinic points, J. Differential Equations, 55, (1984), 225-256.

[19] Rodrigues, H. M. and Silveira, M.: Properties of bounded solutions of linear and nonlinear evolution equations: homoclinics of a beam equation, J. Differential Equations, 70, (1987), 403-440.

[20] Samoilenko, A.M. and Teplinskit, Yu. V.: Countable Systems of Differential Equations, Kiev, 1993 (in Russian).

[21] Shaw, S.W.: Chaotic dynamics of a slender beam rotating about its longitudinal axis, J. Sound Vibr., 124, (1988), 329-343.

[22] Vejvoda, O., Et Al.: Partial Differential Equations: Time-Periodic Solutions, Noordhoff, Groningen, 1981.

[23] Yamaguchi, M.: Existence of periodic solutions of second order nonlinear evolution equations and applications, Funk. Ekvacioj, 38, (1995), 519-538. 